\title{
ON MEROMORPHIC DERIVATIVES
}

\author{
By Wang Yuefei and Wang Shupei
}

\section{Introduction and main results.}

(A) Let $f(z)$ be a transcendental meromorphic function. We use the usual notations of Nevanlinna theory ([3], [8]), then we have the defect relation

$$
\sum_{a \in C} \delta(a, f) \leqq 2 .
$$

The upper bound of two is sharp in general, even not including the infinity. But for the upper bound of the deficiency of derivatives, Yang Lo [9] proved that for $k \geqq 1$,

$$
\sum_{a \in C} \delta\left(a, f^{(k)}\right) \leqq 1+\frac{1}{2 k+1} .
$$

This estimate is better than that in [3] and [5], but the upper bound is still not sharp. There are two problems on the deficiency of derivatives, namely:

( I ) To what extent one can extend the sum of the deficiency of (1.1)?

(II) What is the best upper bound for the left-hand side of (1.1)?

Our first result is an answer to (I).

THEOREM 1. Suppose that $f(z)$ is a transcendental meromorphic function and $k$ is a positive integer. Then we have

$$
\begin{aligned}
& \sum_{a \in C} \delta\left(a, f^{(k)}\right)+\frac{1}{2 k+1} \sum_{b(z)} \delta\left(b(z), f^{(k)}\right)+\frac{1}{2 k+1} \delta\left(\infty, f^{(k)}\right)+\frac{2 k}{2 k+1} \Delta_{k} \\
& \quad \leqq 1+\frac{1}{2 k+1},
\end{aligned}
$$

where the second summation is taken over all the non-constant entire function which is small with respect to $f(z)$, and $\Delta_{k}$ is defined as

$$
\Delta_{k}=\liminf _{r \rightarrow \infty} \frac{N\left(r, 1 / f^{(k+1)}\right)}{T\left(r, f^{(k)}\right)} .
$$

A simple example shows that the inequality is sharp. Let $f(z)=e^{z}$, then for $k \geqq 1$,

Received May 25, 1992; revised February 22, 1993. 


$$
\sum_{a \in C} \delta\left(a, f^{(k)}\right)=\delta\left(0, f^{(k)}\right)=1, \quad \Delta_{k}=0, \quad \sum_{b(z)} \delta\left(b(z), f^{(k)}\right)=0,
$$

and $\delta\left(\infty, f^{(k)}\right)=1$. So the equality of (1.2) holds for this function.

For problem (II), Mues [5] conjectured that the best upper bound for the leftside of (1.1) is one. This conjecture was recently affirmed in [7] and [11] for all integers with at most finitely many exceptions of $k$. In the following theorem we will prove that Mues's conjecture still holds for almost every integers even if we consider the "small" entire functions instead of the complex numbers. In fact, we have

THEOREM 2. Let $f(z)$ be a transcendental meromorphic function. Then we have

$$
\sum_{a(z)} \delta\left(a(z), f^{(k)}\right) \leqq 1
$$

holds for every integer $k$ with at most finitely many exceptional ones, where the summation is taken over all the "small" entire functions of $f(z)$.

(B) The well-known Hayman inequality [2] is an important and striking result for estimating the characteristic function of a meromorphic function. For $k \geqq 1$,

$$
T(r, f)<\left(2+\frac{1}{k}\right) N\left(r, \frac{1}{f}\right)+\left(2+\frac{2}{k}\right) N\left(r,-\frac{1}{f^{(k)}-1}\right)+S(r, f),
$$

where $S(r, f)=o\{T(r, f)\}$, as $r \rightarrow \infty, r \notin e$, and $e$ is a set of finite measure. The small term $S(r, f)$ may not be the same at each occurrence.

In [3], Hayman posed the question: whether the coefficients of $N(r, 1 / f)$ and $N\left(r, 1 / f^{(k)}-1\right)$ in $(1.3)$ are best possible or not. There had no work been done on this problem until 1990. By using a result of Frank and Weissenborn [1], Yang Lo [10] obtained the following result. For $k \geqq 1, \varepsilon>0$,

$$
\begin{aligned}
T(r, f)< & \left(1+\frac{1}{k}\right) N\left(r, \frac{1}{f}\right)+\left(1+\frac{1}{k}\right) N\left(r, \frac{1}{f^{(k)}-1}\right) \\
& -N\left(r, \frac{1}{f^{(k+1)}}\right)+\varepsilon T(r, f)+S(r, f) .
\end{aligned}
$$

When $k$ is large, we see that the coefficients are close to one, which is the best possible coefficients. Our third problem is to consider

(III) Can one extend the complex numbers to small functions of $f(z)$ ? Our Theorem 3 answers the question (III).

THEOREM 3. Let $f(z)$ be a transcendental meromorphic function and $a(z)$ is a small entire function of $f(z)$. Then for any positve integer $k$, positive numbers $\varepsilon$ and $\delta$, there exists a set $E(\delta)$ whose upper logarithmic density does not exceed $\delta$, such that 


$$
\begin{aligned}
T(r, f)< & \left(1+\frac{1}{k}\right) N\left(r, \frac{1}{f}\right)+\left(1+\frac{1}{k}\right) N\left(r, \frac{1}{f^{(k)}-a(z)}\right) \\
& -N\left(r, \frac{1}{\left(f^{(k)} / a\right)^{\prime}}\right)+\varepsilon N(r, f)+S(r, f), \quad r \notin E(\boldsymbol{\delta}) .
\end{aligned}
$$

\section{Lemmas.}

In order to prove our theorems, we need two known results.

LEMMA 1. (Hayman and Miles [4]). Let $f(z)$ be a transcendental meromorphic function and $K$ be a real number $(K>1)$. Then there exists a set $M(K)$ of upper logarithmic density at most

$$
d(K)=\min \left\{\left(2 e^{K-1}-1\right)^{-1},(1+e(K-1)) \exp (e(1-K))\right\}
$$

such that for every positive integer $k$

$$
\varlimsup_{\substack{r \rightarrow \infty \\ r \notin \mathcal{M}(K)}} \frac{T(r, f)}{T\left(r, f^{(k)}\right)} \leqq 3 e K .
$$

Remark. It follows from (2.1) that $d(K) \rightarrow 0$ as $K \rightarrow \infty$.

LEMMA 2. (Milloux inequality [3]). Let $f(z)$ be a transcendental meromorphic function and $a(z)$ be a small meromorphic function of $f(z)$. Then for any integer $k \geqq 1$,

$$
T(r, f)<\bar{N}(r, f)+N\left(r, \frac{1}{f}\right)+N\left(r, \frac{1}{f^{(k)}-a(z)}\right)-N\left(r, \frac{1}{\left(f^{(k)} / a\right)^{\prime}}\right)+S(r, f) .
$$

For convenience, we introduce the following definition.

DEFINITION 1. Let $f(z)$ and $a(z)$ be two meromorphic functions in the complex plane. We say $a(z)$ is a quasi-small function of $f(z)$, if for any $\delta>0$ there exists a set $E(\delta)$ whose upper logarithmic density does not exceed $\delta$, such that

$$
T(r, a)=o\{T(r, f)\}
$$

as $r \rightarrow \infty, r \notin E(\boldsymbol{\delta})$.

LEMMA 3. Suppose that $f(z)$ is a transcendental meromorphic function and $a(z)$ is a small entire function of $f(z)$. Then for every positive integer $k, a^{(-k)}(z)$ is a quasi-small function of $f(z)$ (where $a^{(-k)}(z)$ is the $k^{\text {th }}$ primitive function of $a(z))$.

Proof. By using Lemma 1 on $a^{(-k)}(z)$, we have for $K>1$,

$$
T\left(r, a^{(-k)}\right) \leqq 4 e K T(r, a)
$$


as $r \rightarrow \infty, r \notin M(K)$, where $M(K)$ is a set of upper logarithmic density at most $d(K)$ as in (2.1).

Since $a(z)$ is the small function of $f(z)$, then it follows from (2.2) that

$$
T\left(r, a^{(-k)}\right)=o\{T(r, f)\} \quad \text { as } r \longrightarrow \infty, r \notin M(K) .
$$

On the other hand, we can see from Lemma 1 that $d(K) \rightarrow 0$ as $K \rightarrow \infty$. So, for any given $\delta>0$, we choose a suitable number $K_{0}>1$, such that $d\left(K_{0}\right)<\delta$. Now set $E(\delta)=M\left(K_{0}\right)$, and the conclusion of Lemma 3 follows.

Suppose now that $a_{1}(z), a_{2}(z), \cdots, a_{n}(z)$ are $n$ linearly independent small functions of $f(z)$. Put

$$
L(f)=\frac{W\left(a_{1}, a_{2}, \cdots, a_{n}, f\right)}{W\left(a_{1}, a_{2}, \cdots, a_{n}\right)}
$$

where $W\left(f_{1}, f_{2}, \cdots, f_{n}\right)$ denotes the Wronskian of $f_{1}, f_{2}, \cdots, f_{n}$.

Bearing on the ideas of Frank and Weissenborn [1], we now establish our principal lemma concerning the lower bound of the zeros of the linear differential polynomial $L\left(f^{(k)}\right)$.

LEMMA 4. Let $f(z)$ be a transcendental meromorphic function. Suppose that $a_{1}(z), a_{2}(z), \cdots, a_{n}(z)$ are $n$ linearly independent small entire function of $f(z)$, then for any non-negative integer $k$, positive numbers $\varepsilon$ and $\delta$, there exists a set $E(\delta)$ whose upper logarithmic density does not exceed $\delta$ such that

$$
\begin{gathered}
(k+n) \bar{N}(r, f) \leqq N\left(r, \frac{1}{L\left(f^{(k)}\right)}\right)+(1+\varepsilon) N(r, f)+o\{T(r, f)\}, \\
r \longrightarrow \infty, \quad r \notin E(\boldsymbol{\delta}) .
\end{gathered}
$$

Proof. Since $a_{j}(z)(j=1,2, \cdots, n)$ is entire and

$$
W\left(1, z, \cdots, \frac{1}{(k-1) !} z^{k-1}, a_{1}^{(-k)}, \cdots, a_{n}^{(-k)}\right)=W\left(a_{1}, a_{2}, \cdots, a_{n}\right) \not \equiv 0,
$$

then

$$
\begin{aligned}
L\left(f^{(k)}\right) & =\frac{W\left(a_{1}, a_{2}, \cdots, a_{n}, f^{(k)}\right)}{W\left(a_{1}, a_{2}, \cdots, a_{n}\right)} \\
& =\frac{W\left(1, z, \cdots,(1 /(k-1) !) z^{k-1}, a_{1}^{(-k)}, \cdots, a_{n}^{(-k)}, f\right)}{W\left(1, z, \cdots,(1 /(k-1) !) z^{k-1}, a_{1}^{(-k)}, \cdots, a_{n}^{(-k)}\right)} \\
& :=L_{1}(f), \quad \text { say. }
\end{aligned}
$$

By Lemma 3 , for every $a_{j}^{(-k)}(1 \leqq j \leqq n)$, there exists a set $E_{j}^{1}(\delta)$ whose upper logarithmic density does not exceed $\delta / n$, such that

$$
T\left(r, a_{j}^{(-k)}\right)=o\{T(r, f)\}, \quad r \longrightarrow \infty, \quad r \notin E_{\jmath}^{1}(\delta) .
$$

Set $E_{1}(\delta)=\cup_{j=1}^{n} E_{j}^{1}(\delta)$, then the upper logarithmic density of $E_{1}(\delta)$ does not 
exceed $\delta$ and for every $1 \leqq j \leqq n$.

$$
T\left(r, a_{j}^{(-k)}\right)=o\{T(r, f)\}, \quad r \longrightarrow \infty, \quad r \notin E_{1}(\delta) .
$$

Hence $1, z, \cdots,(1 /(k-1) !) z^{k-1}, a_{1}^{(-k)}, \cdots, a_{n}^{(-k)}$ are $k+n$ linear independent functions which are quasi-small with respect to $f(z)$, and we denote them by $\beta_{1}, \beta_{2}, \cdots, \beta_{k+n}$ respectively.

For $s \geqq 1$, we consider the $C$-linear space $H_{8}$ spanned by the functions $\Pi_{v=1}^{s} \beta j_{v}, j_{v} \in\{1, \cdots, k+n\}$. As in [6], let $d_{s}$ denote the dimension of $H_{s}$, we have

$$
\inf _{s} \frac{d_{s+1}}{d_{s}} \leqq 1
$$

Therefore, for any given $\varepsilon>0$, we can choose an $s_{0}$ such that

$$
\frac{d_{8_{0}+1}}{d_{s_{0}}} \leqq 1+\varepsilon
$$

Let $b_{1}, \cdots, b_{l}\left(l=d_{s_{0}}\right)$ be a basis of $H_{s_{0}}$ and $B_{1}, \cdots, B_{m}\left(m=d_{s_{0}+1}\right)$ be a basis of $H_{s_{0}+1}$. We define $P(f)$ and $A$ by

and

$$
P(f)=W\left(B_{1}, \cdots, B_{m}, b_{1} f, \cdots, b_{l} f\right)
$$

$$
A=\frac{P(f)}{\left(L_{1}(f)\right)^{l}} .
$$

First of all, we may apply the following identity for Wronskians

$$
\begin{aligned}
& W\left(h_{1}, \cdots, h_{m}, g_{1}, \cdots, g_{l}\right)\left(W\left(h_{1}, \cdots, h_{m}\right)\right)^{l-1} \\
& \quad=W\left(W\left(h_{1}, \cdots, h_{m}, g_{1}\right), \cdots, W\left(h_{1}, \cdots, h_{m}, g_{l}\right)\right)
\end{aligned}
$$

to show that

$$
m(r, A)=o\{T(r, f)\}+S(r, f), \quad r \longrightarrow \infty, \quad r \notin E_{1}(\delta) .
$$

Putting $M_{j}(f)=W\left(B_{1}, \cdots, B_{m}, b, f\right), j=1,2, \cdots, l$, we obtain

$$
P(f)=\frac{W\left(M_{1}(f), \cdots, M_{l}(f)\right)}{\left(W\left(B_{1}, \cdots, B_{m}\right)\right)^{l-1}} .
$$

Note that each $M$, is a linear differential operator with

therefore

$$
M_{j}\left(\beta_{\mu}\right)=0, \quad \text { for } \mu=1,2, \cdots, k+n,
$$

$$
M_{j}(f)=N_{j}\left(L_{1}(f)\right),
$$

where again $N_{3}$ is a linear differential operator. Using the well-known lemma on the proximity function of a meromorphic function ([3] or [8]), we have 


$$
m\left(r, \frac{M_{j}(f)}{L_{1}(f)}\right)=S\left(r, L_{1}(f)\right), \quad r \longrightarrow \infty, \quad r \notin E_{1}(\delta) .
$$

Since $L_{1}(f)=L\left(f^{(k)}\right)$ and

$$
\begin{gathered}
T\left(r, f^{(k)}\right) \leqq(k+1) T(r, f)+m\left(r, \frac{f^{(k)}}{f}\right), \\
m(r, A)=m\left(r, \frac{P(f)}{\left(L_{1}(f)\right)^{l}}\right)=S(r, f)+o\{T(r, f)\}, \quad r \longrightarrow \infty, \quad r \notin E_{1}(\delta) .
\end{gathered}
$$

Therefore, by the first fundamental theorem we have

$$
\begin{aligned}
0 \leqq m\left(r, \frac{1}{A}\right)=N(r, A)-N\left(r, \frac{1}{A}\right)+m(r, A)+O(1) \\
\leqq N(r, P(f))+l N\left(r, \frac{1}{L_{1}(f)}\right)-l N\left(r, L_{1}(f)\right)+m(r, A)+O(1), \quad r \rightarrow \infty .
\end{aligned}
$$

Since

and

$$
\begin{aligned}
P(f) & =W\left(B_{1}, \cdots, B_{m}, b_{1} f, \cdots, b_{l} f\right) \\
& =f^{m+l} W\left(\frac{B_{1}}{f}, \cdots, \frac{B_{m}}{f}, b_{1}, \cdots, b_{l}\right), \\
N\left(r, b_{j}\right) & \leqq T\left(r, b_{j}\right)=o\{T(r, f)\},
\end{aligned}
$$

$$
N\left(z, B_{j}\right) \leqq T\left(r, B_{j}\right)=o\{T(r, f)\}, \quad r \longrightarrow \infty, \quad r \notin E_{1}(\delta),
$$

we have

$$
N(r, P(f)) \leqq(m+l) N(r, f)+o\{T(r, f)\}, \quad r \longrightarrow \infty, \quad r \notin E_{1}(\delta) .
$$

On the other hand

$$
\begin{aligned}
N\left(r, L_{1}(f)\right) & =N\left(r, f^{(k+n)}\right)+o\{T(r, f)\} \\
& =N(r, f)+(k+n) \bar{N}(r, f)+o\{T(r, f)\}, \quad r \longrightarrow \infty, \quad r \notin E_{1}(\boldsymbol{\delta}) .
\end{aligned}
$$

From (2.4), (2.5) and (2.6), we have for $r \rightarrow \infty, r \notin E_{1}(\delta)$,

$$
\begin{aligned}
0 \leqq & (m+l) N(r, f)+l N\left(r, \frac{1}{L_{1}(f)}\right)-l N(r, f)-l(k+n) \bar{N}(r, f) \\
& +m(r, A)+o\{T(r, f)\} .
\end{aligned}
$$

Therefore, for $r \rightarrow \infty, r \notin E_{1}(\delta)$, 


$$
\begin{aligned}
(k+n) \bar{N}(r, f) & \leqq \frac{m}{l} N(r, f)+N\left(r, \frac{1}{L\left(f^{(k)}\right)}\right)+S(r, f)+o\{T(r, f)\} \\
& \leqq N\left(r, \frac{1}{L\left(f^{(k)}\right)}\right)+(1+\varepsilon) N(r, f)+o\{T(r, f)\}+S(r, f) .
\end{aligned}
$$

Since $S(r, f)=o\{T(r, f)\}, r \rightarrow \infty, r \notin e$, where $e$ is a set of finite measure, we set $E(\delta)=E_{1}(\delta) \cup e$, hence the upper logarithmic density of $E(\delta)$ does not exceed $\delta$.

This completes the proof of the Lemma 4.

\section{Proof of the theorems.}

Proof of Theorem 1. Let $b_{i}(z)(i=1, \cdots, l)$ be $l$ distinct small entire functions, which can be complex numbers, with respect to $f(z)$. Without loss of generality, we assume that $b_{i}(z)(i=1, \cdots, q ; q \leqq l)$ are the largest linearly independent group in $\left\{b_{i}(z)\right\}^{l}{ }_{i=1}$.

Let $F(z)=\sum_{\imath=1}^{l} 1 /\left(f^{(k)}(z)-b_{i}(z)\right)$, then $([8])$

$$
\sum_{i=1}^{l} m\left(r, \frac{1}{f^{(k)}(z)-b_{i}(z)}\right) \leqq m(r, F)+S\left(r, f^{(k)}\right) .
$$

Denote $L\left(f^{(k)}\right)=W\left(b_{1}, \cdots, b_{q}, f^{(k)}\right) / W\left(b_{1}, \cdots, b_{q}\right)$. By Lemma 4 , we have

$$
(q+k) \bar{N}(r, f)<N\left(r, \frac{1}{L\left(f^{(k)}\right)}\right)+(1+\varepsilon) N(r, f)+o\{T(r, f)\}, \quad r \rightarrow \infty, r \notin E(\delta) .
$$

Noting that $L\left(f^{(k)}\right)=L\left(f^{(k)}-b_{i}\right)(i=1, \cdots, l)$, hence we get

$$
\begin{aligned}
& \sum_{i=1}^{l} m\left(r, \frac{1}{f^{(k)}-b_{i}}\right) \leqq m\left(r, \frac{1}{L\left(f^{(k)}\right)}\right)+\sum_{i=1}^{l} m\left(r, \frac{L\left(f^{(k)}-b_{i}\right)}{f^{(k)}-b_{i}}\right)+S(r, f) \\
& \quad \leqq T\left(r, L\left(f^{(k)}\right)\right)-N\left(r, \frac{1}{L\left(f^{(k)}\right)}\right)+S(r, f) \\
& \quad \leqq m\left(r, f^{(k)}\right)+N\left(r, f^{(k)}\right)+q \bar{N}(r, f)-N\left(r, \frac{1}{L\left(f^{(k)}\right)}\right)+S(r, f) \\
& \quad \leqq m\left(r, f^{(k)}\right)+(2+\varepsilon) N(r, f)+S(r, f)+o\{T(r, f)\}, \quad r \rightarrow \infty, \quad r \notin E(\delta) .
\end{aligned}
$$

Therefore

$$
\begin{aligned}
& m\left(r, f^{(k)}\right)+\sum_{i=1}^{l} m\left(r, \frac{1}{f^{(k)}(z)-b_{i}(z)}\right) \\
& \quad \leqq(2+\varepsilon) T\left(r, f^{(k)}\right)-2 k \bar{N}(r, f)+S(r, f)+o\{T(r, f)\}, \quad r \rightarrow \infty, \quad r \notin E(\boldsymbol{\delta}) .
\end{aligned}
$$

On the other hand, for $p$ finite distinct complex numbers $\left\{a_{j}\right\}_{j=1}^{p}$, 


$$
\begin{aligned}
\sum_{j=1}^{p} m & \left(r, \frac{1}{f^{(k)}-a_{\jmath}}\right) \leqq m\left(r, \frac{1}{f^{(k+1)}}\right)+S\left(r, f^{(k)}\right) \\
& =T\left(r, f^{(k+1)}\right)-N\left(r, \frac{1}{f^{(k+1)}}\right)+S(r, f) \\
& \leqq T\left(r, f^{(k)}\right)+\bar{N}(r, f)-N\left(r, \frac{1}{f^{(k+1)}}\right)+S(r, f) .
\end{aligned}
$$

Therefore, from (3.1) and (3.2), we have

$$
\begin{aligned}
& 2 k \sum_{j=1}^{p} m\left(r, \frac{1}{f^{(k)}-a_{\jmath}}\right)+\sum_{\jmath=1}^{l} m\left(r, \frac{1}{f^{(k)}-b_{j}(z)}\right)+m\left(r, f^{(k)}\right) \\
& \leqq(2 k+2+\varepsilon) T\left(r, f^{(k)}\right)-2 k N\left(r, \frac{1}{f^{(k+1)}}\right) \\
&+S(r, f)+o\{T(r, f)\}, \quad r \longrightarrow \infty, \quad r \notin E(\boldsymbol{\delta}) .
\end{aligned}
$$

However, from Lemma 1 it follows that

$$
T(r, f) \leqq 4 e K T\left(r, f^{(k)}\right), \quad r \longrightarrow \infty, \quad r \notin M(K) .
$$

Noting that $S(r, f)=o\{T(r, f)\}, r \rightarrow \infty, r \notin e$ and $e$ is a set of finite measure, we have from (3.3) that

$$
\sum_{i=1}^{l} \delta\left(b_{i}(z), f^{(k)}\right)+2 k \sum_{\jmath=1}^{p} \delta\left(a_{\jmath}, f^{(k)}\right)+\delta\left(\infty, f^{(k)}\right)+2 k \Delta_{k} \leqq 2 k+2+\varepsilon .
$$

Letting $l, p \rightarrow \infty, \varepsilon \rightarrow 0$, we get

$$
2 k \sum_{a \in C} \delta\left(a, f^{(k)}\right)+\sum_{b(z)} \delta\left(b(z), f^{(k)}\right)+\delta\left(\infty, f^{(k)}\right)+\Delta_{k} \cdot 2 k \leqq 2 k+2,
$$

where the second summation is taken over all the small entire functions including finite complex numbers.

This completes the proof of Theorem 1 .

Remark. In fact, from (3.3) we can get the following.

COROLLARY 1. If $f(z)$ is meromorphic and transcendental, then

$$
\sum_{a(z)} \delta\left(a(z), f^{(k)}\right)+\delta\left(\infty, f^{(k)}\right)+2 k \sum_{a \in C} \Theta\left(a, f^{(k)}\right) \leqq 2 k+2,
$$

where the first summation is taken over all the small entire functions, including finite complex numbers and

$$
\Theta\left(a, f^{(k)}\right)=1-\varlimsup_{r \rightarrow \infty} \frac{\bar{N}\left(r, f^{(k)}=a\right)}{T\left(r, f^{(k)}\right)} .
$$

Proof of Theorem 2. Let $a_{1}(z), \cdots, a_{q}(z)$ be $q$ arbitrary distinct small entire 
functions. Without loss of generality, we assume that $a_{1}(z), \cdots, a_{n}(z),(n \leqq q)$ are the largest linear independent group in $\left\{a_{1}(z)\right\}_{j=1}^{q_{j=1}}$. Put

Since $([8])$

$$
L\left(f^{(k)}\right)=\frac{W\left(a_{1}, a_{2}, \cdots, a_{n}, f^{(k)}\right)}{W\left(a_{1}, a_{2}, \cdots, a_{n}\right)} .
$$

$$
\begin{aligned}
\sum_{j=1}^{q} m\left(r, \frac{1}{f^{(k)}-a_{\jmath}}\right) & \leqq \sum_{j=1}^{q} m\left(r, \frac{L\left(f^{(k)}-a_{j}\right)}{f^{(k)}-a_{\jmath}}\right)+m\left(r, \frac{1}{L\left(f^{(k)}\right)}\right)+O(1) \\
& \leqq T\left(r, L\left(f^{(k)}\right)\right)-N\left(r, \frac{1}{L\left(f^{(k)}\right)}\right)+S\left(r, f^{(k)}\right) \\
N\left(r, L\left(f^{(k)}\right)\right) & =N\left(r, f^{(k)}\right)+n \bar{N}(r, f)+S(r, f),
\end{aligned}
$$

and

$$
m\left(r, L\left(f^{(k)}\right)\right) \leqq m\left(r, f^{(k)}\right)+S\left(r, f^{(k)}\right) \leqq m\left(r, f^{(k)}\right)+S(r, f),
$$

we have

$$
\sum_{j=1}^{q} m\left(r, \frac{1}{f^{(k)}-a_{\jmath}}\right) \leqq T\left(r, f^{(k)}\right)+n \bar{N}(r, f)-N\left(r, \frac{1}{L\left(f^{(k)}\right)}\right)+S(r, f) .
$$

We distinguish two cases.

(i) There exists an integer sequence $\left\{k_{j}\right\}_{j=1}^{\infty}$, such that

$$
N\left(r, \frac{1}{L\left(f^{\left(k_{j}\right)}\right)}\right) \leqq n \bar{N}(r, f)
$$

holds on $\boldsymbol{R}$ except possibly a set $\varepsilon_{k_{j}}$ of finite measure.

In this case, from Lemma 4 , we have for any positive numbers $\varepsilon$ and $\delta$,

$$
\left(k_{j}+n\right) \bar{N}(r, f) \leqq N\left(r, \frac{1}{L\left(f^{\left(k_{j}\right)}\right)}\right)+(1+\varepsilon) N(r, f)+o\{T(r, f)\}, r \rightarrow \infty, r \notin E_{k_{j}}(\boldsymbol{\delta}) .
$$

where $E_{k_{j}}(\boldsymbol{\delta})$ is a set whose upper logarithmic density does not exceed $\delta$. Therefore,

$$
k_{j} \bar{N}(r, f) \leqq(1+\varepsilon) N(r, f)+o\{T(r, f)\}, \quad r \longrightarrow \infty, \quad r \notin E_{k_{j}}(\delta) \cup e_{k_{j}} .
$$

For any given $\boldsymbol{\varepsilon}^{\prime}>0$, we choose an $k_{\rho_{0}}$ such that

$$
\varepsilon^{\prime} k_{j_{0}}>(1+\varepsilon) n \text {. }
$$

Then

$$
n \bar{N}(r, f)<\varepsilon^{\prime} N(r, f)+o\{T(r, f)\}, \quad r \longrightarrow \infty, \quad r \notin E_{0},
$$

where $E_{0}=E_{k_{j 0}}(1 / 4) \cup e_{k_{j 0}}$, whose upper logarithmic density does not exceed $1 / 4$.

By Lemma 1, we can choose some $K$, such that for every integer $k$ 


$$
T(r, f)<4 e K T\left(r, f^{(k)}\right), \quad r \longrightarrow \infty, \quad r \notin M(K),
$$

where $M(K)$ is a set of upper logarithmic density at most $1 / 4$.

Put $E_{1}=E_{0} \cup M(K)$. Then the upper logarithmic density of $E_{1}$ is at most $1 / 2$. From (3.5) and (3.6), we have for every positive integer $k$,

$$
n \bar{N}(r, f)<\varepsilon^{\prime} N(r, f)+o\left\{T\left(r, f^{(k)}\right)\right\}, \quad r \longrightarrow \infty, \quad r \notin E_{1} .
$$

We can now deduce from (3.4) and (3.7) that for every positive integer $k$,

$$
\begin{gathered}
\sum_{\jmath=1}^{q} m\left(r, \frac{1}{f^{(k)}-a_{\jmath}}\right) \leqq T\left(r, f^{(k)}\right)+\varepsilon^{\prime} N(r, f)+o\left\{T\left(r, f^{(k)}\right)\right\}+S(r, f), \\
r \longrightarrow \infty, \quad r \notin E_{1} .
\end{gathered}
$$

Since $S(r, f)=o\{T(r, f)\}, r \rightarrow \infty, r \notin e$, and $e$ is a set of finite measure, we have from (3.6), $S(r, f)=o\left\{T\left(r, f^{(k)}\right)\right\}, r \rightarrow \infty, r \notin M(K) \cup e$. Therefore,

$$
\sum_{j=1}^{q} \delta\left(a_{\jmath}, f^{(k)}\right) \leqq 1+\varepsilon^{\prime} .
$$

Thus for every positive integer $k$, we obtain

$$
\sum_{a(z)} \delta\left(a(z), f^{(k)}\right) \leqq 1
$$

by letting $\varepsilon^{\prime} \rightarrow 0$ and $q \rightarrow \infty$.

(ii) For every positive integer $k$,

$$
n \bar{N}(r, f) \leqq N\left(r, \frac{1}{L\left(f^{(k)}\right)}\right)
$$

holds on a set $S_{k}$ of infinite measure, except possibly finitely finitely many integers $k_{1}, \cdots, k_{m}$. Then we have from (3.4) and (3.8) that

$$
\sum_{j=1}^{q} m\left(r, \frac{1}{f^{(k)}-a_{\jmath}}\right) \leqq T\left(r, f^{(k)}\right)+S(r, f), \quad r \subseteq S_{k} .
$$

Therefore, again by applying Lemma 1 to $S(r, f)$ we have

$$
\sum_{\jmath=1}^{q} \delta\left(a_{\jmath}, f^{(k)}\right) \leqq 1
$$

for every $k \notin\left\{k_{j}\right\}_{j=1}^{m}$. This completes the proof of Theorem 2 .

Proof of Theorem 3. Let $n=1$ in Lemma 4 and

$$
L\left(f^{(k)}\right)=\frac{W\left(a, f^{(k)}\right)}{a} .
$$

Then for $r \notin E(\delta), r \rightarrow \infty$ 


$$
(k+1) \bar{N}(r, f)<(1+\varepsilon) N(r, f)+N\left(r, \frac{1}{L\left(f^{(k)}\right)}\right)+o\{T(r, f)\} .
$$

From Lemma 2

$$
T(r, f)<\bar{N}(r, f)+N\left(r, \frac{1}{f}\right)+N\left(r, \frac{1}{f^{(k)}-a}\right)-N\left(r, \frac{1}{\left(f^{(k)} / a\right)^{\prime}}\right)+S(r, f) .
$$

Noting that

$$
L\left(f^{(k)}\right)=\frac{a f^{(k+1)}-a^{\prime} f^{(k)}}{a}=a\left(\frac{f^{(k)}}{a}\right)^{\prime}
$$

hence, from (3.9) and (3.10), we have

$$
k \bar{N}(r, f) \leqq N\left(r, \frac{1}{f}\right)+N\left(r, \frac{1}{f^{(k)}-a}\right)+\varepsilon N(r, f)+S(r, f), \quad r \rightarrow \infty, \quad r \notin E(\boldsymbol{\delta}),
$$

where we write $o\{T(r, f)\}$ as $S(r, f)$. For $r \notin E(\delta), r \rightarrow \infty$,

$$
\begin{aligned}
T(r, f) & <\left(1+\frac{1}{k}\right) N\left(r, \frac{1}{f}\right)+\left(1+\frac{1}{k}\right) N\left(r, \frac{1}{f^{(k)}-a}\right) \\
& -N\left(r, \frac{1}{\left(f^{(k)} / a\right)^{\prime}}\right)+\varepsilon N(r, f)+S(r, f)
\end{aligned}
$$

follows from (3.10) and (3.11), which completes the proof of Theorem 3.

Remark. The method used in this paper is effective in dealing with the problems of meromorphic derivatives, specially concerning "small entire functions". Whether the "small entire functions" in our theorems can be replaced by "small meromorphic functions" is still a problem. The difficulty is that the existence of the primitive functions of the small entire functions is needed in the proof of our principal lemma.

Acknowledgement. The authors would like to thank Professor Yang Lo for his suggestions and comments.

\section{REFERENCES}

[1] Frank, G. AND Weissenborn, G., On the zeros of linear differential polynomails of meromorphic functions, Complex Variables 12 (1989), 77-81.

[2] HaYman, W.K., Picard values of meromorphic functions and their derivatives, Ann. of Math. 70 (1959), 9-42.

[3] Hayman, W.K., Meromorphic functions, Clarendon Press, Oxford, 1964.

[4] Hayman, W.K. AND Miles, J., On the growth of a meromorphic function and its derivatives, Complex Variables 12 (1989), 245-260. 
[5] MuEs, E., Über eine Defekt und Verzweigungsration für die Ableitung Meromorpher Funktionen, Manuscripta Math. 5 (1971), 275-297.

[6] Steinmetz, N., Eine Verallgemeinerung des zweiten Nevanlinnaschen Haupsatzes, J. Reine Angew. Math. 368 (1986), 134-141.

[7] Wang Yuefei, On Mues conjecture and Picard values, to appear in Science in China.

[8] YANG Lo, Value distribution theory and its new researches, Beijing, 1982.

[9] YANG Lo, Precise estimate of total deficiency of meromorphic derivatives, J. Analyse Math. 55 (1990), 287-296.

[10] Yang Lo, Precise fundamental inequalities and sum of deficiencies, Science in China Ser. A 34 (1991), 157-165.

[11] Yang Lo AND Wang Yuefei, Drasin's problems and Mues conjecture, to appear in Science in China.

Institute of Mathematics,

Academia Sinica,

BEIJING 100080,

CHINA

Department of Mathematics,

UNIVERSITY OF JOENSUU,

P.O. Box 111,

SF-80101 JOENSUU,

FinLAND 